\title{
Antibiotic resistance profiles from isolated bacteria in outpatient infertility clinic in Greece
}

\author{
Karakalpakis D , Kostaras K1, Asonitis K², Dimitriadi D³ ,Pittaras $\mathrm{T}^{4}$, Charvalos $\mathrm{E}^{2,3}$, \\ Daikos $\mathrm{G}^{4}$, Mantzourani $\mathrm{M}^{4}$, Pappa $\mathrm{K}^{4}$
}

${ }^{1}$ Institute of Life (IOL) IASO, Athens, Greece, ${ }^{2}$ Central Laboratories IASO Hospital, Athens, Greece, ${ }^{3}$ InVitroLabs, SA. Athens, Greece, ${ }^{4} 1$ st Department of Obstetrics and Gynecology, Alexandra Hospital, National \& Kapodistrian University of Athens, Athens, Greece

\author{
Correspondence \\ Dr Ekatherina Charvalos, directorcentralabs@iaso.gr
}

\section{Abstract}

Aim: To investigate the prevalence of common aerobic gram positive and gram negative bacteria, Mycoplasma hominis, Ureaplasma urealyticum and Chlamydia trachomatis in symptomatic and asymptomatic Greek patients and to determine antibiotic resistance profiles. Methods: This retrospective study included a total of 316 adult men examined at the Assisted Reproduction Department of IASO- Obstetrics and Gynecology clinic in Athens, Greece. Sperm have been collected and proceed to culture and antibiotic sensitivity at the Central Laboratories following a standard protocol. Results: Twelve inappropriate out of 316 samples were excluded from the study. Out of the remaining 304 sperm samples 111 (37.5 \%) were positive. Antibiotic sensitivity testing detected resistances to some commonly used antibiotics such as b-lactams and the quinolones. Ureaplasma urealyticum and Mycoplasma hominis were the most frequently isolated bacteria (45\%), followed by Enterobacteriaceae (40\%) and Enterococci 12.6\%. The majority of 45 Enterobacteriaceae isolates, were Escherichia coli (31 strains $/ 68 \%$ ) corresponding to $27.9 \%$ of the total number of positive cultures. One infection to Chlamydia trachomatis was detected by an immunochromatic rapid test, one Candida sp, one Pseudomonas aeruginosa, two M. hominis and three Streptococcus group B. Escherichia coli were resistant to b lactams in about 38.7\% due to b-lactamase, and $22.5 \%, 9.6 \%, 6.4 \%$, were resistant to nitrofurantoin, sulfamethoxazole and ciprofloxacin respectively. Enterococci have shown resistance due to b-lactamase and PBP 5 alteration/hyperproduction. Ureaplasmas were resistant to the fluoroquinolones tested ciprofloxacin and ofloxacin, at $72.2 \%$ and $62.3 \%$ respectively. Conclusion: Carriage of bacteria in sperm is controversial for its contribution in sperm quality and fertility. In our IVF unit, we follow a protocol of isolation and antibiotic profiling of bacteria from sperm culture regardless of their concentration in sperm and giving the numbers/ml. This helps doctors to distinguish carriage or infection and to decide about potential therapy. Given the antibiotic resistances shown by this study, the importance of culture against empiric therapy in assisted reproduction patients is also clearly demonstrated.

Key words: assisted reproduction techniques (ART), In vitro fertilization (IVF), Enterobacteriaceae, Mycoplasmas, men, antibiotic resistance, infertility 


\section{Introduction}

Male infertility is a complex problem which affects approximately $5 \%$ of male population. Body of knowledge of the etiology of infertility remains unknown $^{1,2}$. Genital infection can impair the quality of spermatozoa and negatively affect male fertility ${ }^{3}$.

Semen culture for the detection of bacteria is usually accomplished together with sperm analysis. It has been shown recently that presence of bacteria in semen can affect sperm quality ${ }^{4}$, although some controversies still exist. In most cases those bacteria originate from the urinary tract of patients or can be sexually transmitted infections caused by several pathogens and representing a public health problem associated with fertility ${ }^{5}$. Aerobic gram negative, Mycoplasmas, Ureaplasmas and Chlamydia are the most frequently isolated bacteria from semen.

Gram negative bacteria, Enterobacteriaceae with E. coli as the predominant strain are frequently isolated from sperm; its negative influence on human spermatozoa $^{6}$ and sperm quality ${ }^{7}$ is by far recognized.

Gram-positive uropathogenic bacteria influence on sperm morphology and function has been poorly investigated until now. Among them, enterococci are the most frequently isolated bacteria. Body of knowledge on its pathogenic effect on sperm is unclear. However it was recently shown that some virulence factors of enterococci may affect sperm functions [8]. Mycoplasmas, Ureaplasmas and Chlamydia trachomatis may colonize the male urethra and they represent potentially pathogenic species playing an etiologic role in male infertility $9,10,11,12$.

The clinical importance of bacteria in semen is significant if paired with signs of infection. A common practice in assisted reproduction departments the administration of an appropriate antibiotic treatment after culture, isolation, identification and antibiotic resistance determination. Some others choose empiric antibiotic administration, but this might raise antibiotic resistance.

The aim of this study was to investigate the semen contamination and antibiotic resistance profiles of common bacteria and mycoplasmas in our assisted reproduction clinic.

\section{Subjects, materials and methods}

\section{Patient population and sample collection}

A total of 316 adult men examined at the Assisted Reproduction Department of IASO- Obstetrics and Gynecology clinic in Athens, Greece for sperm quality and bacterial infection from 01/04/2014 to $30 / 05 / 2015$. IASO is one of the biggest Maternity clinics in Europe, with 12000 childbirths/year. Semen has been collected by masturbation after 3-5 days of sexual abstinence. Semen samples were collected by masturbation. Patients were asked to urinate, wash the hands, penis and scrotum before ejaculation to avoid possible contamination from external genital system or urine. Samples proceeded immediately for examination at the embryology laboratory for sperm quality and at the microbiology department of the hospital for bacterial culture and antibiotic sensitivity. Totally 12 samples were excluded from the study for collecting samples inappropriately or receiving antibiotic therapy in the previous 20 days.

\section{Bacterial culture and antibiotic sensitivity testing}

Semen collected for bacterial culture have been tested by light microscopy for bacteria, white and red blood cells and plated on MacConkey agar No 2, Blood agar, Mannitol salt agar, Thayer Martin, Sabouraud agar and Mueller Hinton agar (Biomérieux, France) and Chromagar Strep B (Bioprepare, Papanikolaou \& Sofou Co, Greece). Antibiotic sensitivity testing for common aerobic bacteria was performed by the Kirby Bauer method according to CLSI standards. 
Samples were inoculated using a calibrated loop on agar plates, which were incubated overnight at $37^{\circ} \mathrm{C}$ in normal air or with $5 \% \mathrm{CO}^{2}$. The microorganisms were identified by gram stain, oxidase, catalase and other biochemical tests using Bio-Mérieux products (Bio-Mérieux, Marcy L'Etoile, Lyon, France).

\section{Detection of mycoplasmas and testing against antibiotics}

Briefly, clinical samples were collected with a swab in Urea-Arginine LYO 2 broth (BioMérieux) containing $3 \mathrm{ml}$ of genital mycoplasma transport medium. They were immediately sent to the laboratory. As soon as they arrived, the samples were inoculated in Mycoplasma IST 2 (BioMérieux), to test for viability and antibiotic sensitivity. Susceptibility was tested against 9 antibiotics, as follows: doxycycline, tetracycline, erythromycin, clarithrothromycin, ciprofloxacin, josamycin, azithromycin, ofloxacin and pristinamycin. An aliquot of the original sample was inoculated onto A7 agar (Bioprepapre, Papanikolaou \& Sofou Co, Greece) for cultivation of both genital mycoplasmas.

Broth cultures were incubated at $37 \mathrm{oC}$ in aerobic conditions and observed for a $\mathrm{pH}$ change within 48 hours. The A7 agar plates were incubated at $37 \mathrm{oC}$ in an atmosphere of 5\% $\mathrm{CO} 2$ and examined microscopically daily for 5 days for the appearance of typical mycoplasma colonies.

\section{Detection of Chlamydia trachomatis}

Detection of Chlamydia trachomatis was carried out by the Chlamydia Antigen (CHL, BIOSYNEX, Gottingen, Germany), a simple and rapid, immunochromatographic test.

\section{Results}

In this study we excluded $12(3.7 \%)$ out of 316 sperm samples as inappropriate. Therefore among three hundred and four (314) sperm cultures exam- ined, 111 were positive for common aerobic bacteria, Mycoplasma hominis, Ureaplasma urealyticum and Candida spp, representing about $30 \%$ of specimen. We reported all isolated bacteria in sperm regardless of concentration.

Table 1 shows the frequency of isolation of each microorganism of the study. As can be observed the most frequently isolated bacteria were mycoplasmas, followed by Enterobacteriaceae and Enterococci.

Among forty five (45) isolated Enterobacteriacae, thirty one (1) were identified as E coli, six (6) as Proteus mirabilis, three (3) as K. ozoneae, three (3) as K. pneumoniae, and two (2) Citrobacter coseri. Co-isolation of E. coli was observed in two cases, one with K. ozonae and one with Enterococcus faecalis.

About 38.7\% (12/31) of E coli strains have shown a resistance to b-lactam antibiotics penicillisane mediated, $22.5 \%(7 / 31)$ to nitrofurantoin, 9.6\% (3/31) to sulfamethoxazole (SXT), $6.4 \% \quad(1 / 31)$ to ciprofloxacin and to all fluoroquinolones tested 3.2\% (1/31). One Klebsiella sp out of six isolated (16.6\%) was extended spectrum B Lactamase (ESBL), 83\% were resistant to nitrofurantoin and all of the strains were resistant to ampicillin as expected. About one third of Proteus sp strains isolated were resistant to ampicillin and amoxicillin as penicillinase producers and all of them resistant to nitrofurantoin as expected.

About one third of the isolated Enterococci strains were resistant to the erythromycin. Two strains were b-lacatmase producers and two with altered PBP 5. About $7 \%$ of the strains were resistant to doxycycline and ciprofloxacin. There was one isolate characterized as VRE (Vancomycin Resistant Enterococcus). The three Streptococcus B strains were sensitive to all antibiotics tested, (b-lactams, cephalosporins and fluoroquinolones).

Among 48 Ureaplasma strains nine were isolated on agar A7 and not in broth. Those strains were not 
subjected to antibiotic sensitivity because sensitivity is measured in broth medium. Consequently, $37 \mathrm{U}$. urealyticum and two M. hominis were tested against antibiotics. Most of the strains were resistant to the quinolones ciprofloxacin (72.2\%) and ofloxacin $(62.3 \%)$. Five \% of the strains were resistant to the commonly used macrolides, namely erythromycin, roxithromycin and azithromycin. All of the U. urealyticum strains were sensitive and all of M. hominis resistant respectively to Lincomycin.

\section{Discussion}

Infections of the male genital system account for up to $15 \%$ of infertility in men ${ }^{13}$. Acute or chronic bacterial inflammation in male reproductive genital tract may account for sperm cell function, spermatogenetic process and apoptosis ${ }^{4,14,15,16}$. World Health Organization guidelines (WHO, 1992) define genital tract infection in men by the following parameters:

(i) significant bacteriospermia (103 bacteria/ml ejaculate);

(ii) detection of Neisseria gonorrhoeae/C. trachomatis/U. urealyticum;

(iii) significant leukocytospermia (.106 peroxidase-positive leukocytes $/ \mathrm{ml}$ ejaculate). Those parameters are however still under contestation and there are no guidelines to follow in case of bacterial infections in sperm donors. However in most routine laboratories, spermiocultures are traditionally considered positive $\geq 103 \mathrm{CFU} \mathrm{ml}-1$ in case of gram positive cocci and $\geq 104 \mathrm{CFU}$ ml-1 in case of gram negative rods.

According to our protocol regardless of numbers of bacteria all isolated common aerobic strains and their respective antibiotic resistance profiles are reported in the test result. Culture for strict anaerobes was not carried out in this study. Ureaplasmas were the most common bacterial contaminant of sperms followed by Enterobacteriaceae and Enterococci. Escherichia coli were the most frequent species among
Enterobacteriaceae with common antibiotic resistance profile. Strong evidence of the impact of E coli on fertility and sperm quality is proven ${ }^{15,16}$. In a recent in vitro study, Bacteroides ureolyticus demonstrated the most significant negative effect on sperm structure and function, but $\mathrm{E}$ coli also proven as a factor conducting diminished motility and sperm membrane injury of lipid bilayers ${ }^{17}$. Spermagglutinating E. coli in an animal model demonstrated an important factor of infertility ${ }^{18}$. In our Laboratory we consider carefully E coli and Enterobacteriaceae as sperm pathogens and provide the clinician with antibiotic resistant profiles.

Table1. Isolated bacteria from 111 positive

\begin{tabular}{lcc} 
Strain & Number* & \% \\
\hline Enterobacteriaceae & 45 & 40.5 \\
Escherichia coli & $31 / 45$ & 27.9 \\
Proteus spp & $6 / 45$ & 5.4 \\
Klebsiella pneumonieae & $4 / 45$ & 3.6 \\
Klebsiella ozoneae & $2 / 45$ & 1.8 \\
Citrobacter koseri & $2 / 45$ & 1.8 \\
Streptococcus b & 3 & 2.7 \\
Enterococcus spp & 14 & 12.6 \\
U. urealyticum and M. hominis & 50 & 45 \\
U. urealyticum & 48 & 43.3 \\
M. hominis & 2 & 1.8 \\
Candida spp & 1 & 0.9 \\
Pseudomonas aeruginosa & 1 & 0.9 \\
Chlamydia trachomatis & 1 & 0.9 \\
Number of positive cultures & 111 & 36.54 \\
Number of negative cultures & 193 & 63.56 \\
Total number of samples & 304 & 100 \\
\hline
\end{tabular}

*Dual infection was observed in 11 cases: a. U. urealyticum and Chlamydia trachomatis (1), b. U. urealyticum and Candida sp (1), U. urealyticum and E coli (5), U. urealyticum and Klebsiella sp (1), U. urealyticum and Enterococcus (2), M. hominis and U. urealyticum

Compared to other studies Streptococcus group B was isolated in a lesser degree than reported previously $^{19,20}$ and with no resistance to b-lactams the drug of choice in the treatment of the microorganism. Because of its importance not only in genital 
tract but in neonatal infections too we provide information to the couples about "dual infection", transmission and the guidelines on Streptococcus B treatment at 34 week of gestation ${ }^{21}$, if fertilization is successful.

The presence of U. urealyticum and M. hominis was shown in about $45 \%$ of the positive cultures and this finding is similar to other reports ${ }^{22}$. The importance of U. urealyticum and M. hominis in infertility is proven $9,10,11$, although body of knowledge remains fragmentary. As for the antibiotic sensitivity test, we systematically use a commercial microdilution method (see Subjects, Materials and Methods). Results from our study confirm that a high percentage of the microorganisms isolated were resistant to ciprofloxacin and ofloxacin. A recent study in women in North Greece showed also high quinolone resistance rates in Ureaplasmas, ${ }^{23}$ and this might be due to the extended use of quinolones in Greece for the treatment of urinary tract infections. Some of the strains in this study were detected only on agar A7. Consequently, since our commercial antimicrobial testing is a broth microdilution method, we had not the possibility to perform antibiograms to all isolates. This means that the number of quinolone resistant strains might be higher than reported here. Empiric therapy consequently, is unavoidable in such situation.

We did not have the possibility to detect Mycoplasma genitalium and Helicobacter pylori, microorganisms that are associated with genital infections and infertility in men ${ }^{10,22}$. This is due, first, because there are no guidelines about systematic detection of them and second because both of the microoganisms are detected by molecular methods.

There was no N. gonorhoea infection diagnosed among our patients by culture method, microscopy and gram stain. This could be explained by the fact that most of our patients had already visited the clinician at least once and received therapy if symptoms were present, either after laboratory confirmation or with an empiric antibiotic treatment. In addition it is not recommended to screen men at low risk, and our population study group is at low risk.

Similarly, only one Chlamydia trachomatis was detected corresponding to about $0.28 \%$ of the samples tested. Chlamydia trachomatis chronic infection of the male genital tract is associated with infertility ${ }^{24}$. It seems that recognition of chlamydial antigens is associated with Toll-like receptors and other pathogen recognition receptors expressed on epithelial cells and immune cells from the male genital system. This in turn, provokes a chronic inflammation that might orient to the onset of an autoimmune process in genetically susceptible individuals ${ }^{24}$. It seems in addition that the presence of a mild inflammation remains miss-diagnosed in some individuals. We used a rapid immunochromatography test for the detection of chlamydia. According to the literature antigen detecting method(s), as used in our laboratory is/are of a limited sensitivity and Nucleic Acids Amlification Techniques (NAAT) tests are recommended for the detection of Chlamydia ${ }^{25}$. A recent study confirmed the presence and importance of N. gonorrhea, M. genitalium, U. urealyticum and Chlamydia trachomatis in men by using molecular detection ${ }^{26}$. According to this (our) co-authorship, it seems that molecular detection can improve the diagnosis of male genital tract infection and consequently the choice of treatment especially for the above mentioned pathogens.

Finally, Enterococci were present in $12.5 \%$ of the total number of positive cultures. Mehta et al. ${ }^{27} \mathrm{re}-$ ported that Enterococcus faecalis was isolated from $53 \%$ of patients with infertility and noticed that he mean sperm concentration, as well as the mean percentage of morphologically normal spermatozoa, was significantly lower in semen infected with $\mathrm{S}$. faecalis compared with that containing micrococci or alpha-haemolytic streptococci and the uninfected samples. Although not considered as a sexually 
transmitted infection, pathogenicity of Enterococcus spp in men genital tract is by far known ${ }^{4,8,27}$.

In our test result we report the exact number of Enterococci as well as the species to the doctors. Antibiotic resistance profiles of Enterococci, showed resistances to some extent to the commonly used antibiotics in clinical practice including one VRE strain. We have no details on the carrier patient of this strain, with the exception of hospitalization for a routine gastrointestinal operation in the past three years.

Results of this study did not correlate directly sexually transmitted infection and infertility, but depict the profile of strains isolated in the specific men population in our outpatient assisted reproduction clinic. The study also designated the sensitivities to commonly used antibiotics and the benefits of our practice to report all microorganisms isolated regardless of numbers and their susceptibilities.

European Directive on technical requirements for donation, procurement and testing of human tissues and cells designates the methods to clear sperm from bacteria before donation ${ }^{28}$. However, to achieve better quality of sperm cells it is necessary to perform classical bacterial culture and NAAT to detect genital pathogens and treat the patient. Our practice at IASO assisted reproduction clinic showed the importance of culture before donation. Because NAAT techniques can drastically increase sensitivity detection mainly for Chlamydia trachomatis, and help to detect other, difficult to culture bacteria associated with sperm pathogenicity we recently adopted Real Time PCR and recommend to the patient in case of non-fertile men with unknown origin sperm disorders.

\section{References}

1. Dada R, Kumar M, Jesudasan R, Fernández JL, Gosálvez J, Agarwal A. Epigenetics and its role in male infertility. J Assist Reprod Genet 2012; 29:213-23.

2. Poongothai J, Gopenath TS, Manonayaki S. Genet- ics of human male infertility. Singapore Med J 2009; 50:336-47.

3. Pellati D, Mylonakis I, Bertoloni G, Fiore C, Andrisani A, Ambrosini G, et al. Genital tract infections and infertility. Eur J Obstet Gynecol Reprod Biol 2008; 140:3-11.

4. Sanocka-Maciejewska D, Ciupińska M, Kurpisz M. Bacterial infection and semen quality. J Reprod Immunol 2005; 67:51-6.

5. Gimenes F, Souza RP, Bento JC, Teixeira JJ, MariaEngler SS, Bonini MG, Consolaro ME. Male infertility: a public health issue caused by sexually transmitted pathogens. Nat Rev Urol 2014; 11(12):672-87.

6. Diemer T, Huwe P, Michelmann HW, Mayer F, Schiefer HG, Weidner W. Escherichia coli-induced alterations of human spermatozoa. An electron microscopy analysis. Int J Androl 2000; 23:178-86.

7. Boguen R, Treulen F, Uribe P, Villegas JV. Ability of Escherichia coli to produce hemolysis leads to a greater pathogenic effect on human sperm. Fertil Steril 2015; 103(5):1155-61.

8. Qiang H, Jiang MS, Lin JY, He WM. Influence of enterococci on human sperm membrane in vitro. Asian J Androl 2007; 9:77-81.

9. Andrade-Rocha FT. Ureaplasma urealyticum and Mycoplasma hominis in men attending for routine semen analysis. Prevalence, incidence by age and clinical settings, influence on sperm characteristics, relationship with the leukocyte count and clinical value. Urol Int. 2003; 71(4):377-81.

10. Gdoura R, Kchaou W, Chaari C, Znazen A, Keskes L, Rebai T, Hammami A. Ureaplasma urealyticum, Ureaplasma parvum, Mycoplasma hominis and Mycoplasma genitalium infections and semen quality of infertile men. BMC Infect Dis 2007;8(7):129.

11. Liu J, Wang Q, Ji X, Guo S, Dai Y, Zhang Z, Jia L, Shi Y, Tai S, Lee Y. Prevalence of Ureaplasma urealyticum, Mycoplasma hominis, Chlamydia tra- 
chomatis infections, and semen quality in infertile and fertile men in China. Urology 2014;83 (4):795-9.

12. Ljubin-Sternak S, Meštrović T. Chlamydia trachomatis and Genital Mycoplasmas: Pathogens with an Impact on Human Reproductive Health. J Pathog. 2014;2014:183167. doi: 10.1155/ 2014/183167. Epub 2014 Dec 31. Review.

13. Pellati, D, Mylonakis I, bertoloni G, Fiore C, Andrisani A, Ambrisini G et al. Genital tract infections and infertility. Eur J Obstet gynecol Rweprod Biol 2008;140:3-11.

14. Villegas J, Schulz M, Soto L, Sanchez R. Bacteria induce expression of apoptosis in human spermatozoa. Apoptosis 2005;10:105-10.

15. Fraczek M, Wiland E, Piasecka M, Boksa M, Gaczarzewicz D, Szumala-Kakol A, Kolanowski T, Beutin L, Kurpisz M. Biol Reprod. Fertilizing potential of ejaculated human spermatozoa during in vitro semen bacterial infection. Fertil Steril 2013;89(3):59

16. Bozhedomov VA, Semenov AV, Konyshev AV, Lipatova NA, Pacanovskaja GM, Bozhedomova GE, Tret'jakov AV. Reproductive function in men with chronic prostatitis: clinical, disease history and microbiological risk aspects. Urologiia 2015;1: 70-4, 76-8.

17. Lang T, Dechant M, Sanchez V, Wistuba J, Boiani M, Pilatz A, Stammler A, Middendorff R, Schuler G, Bhushan S, Tchatalbachev S, Wübbeling F, Burger M, Chakraborty T, Mallidis C, Meinhardt A. Structural and functional integrity of spermatozoa is compromised as a consequence of acute uropathogenic E. coli-associated epididymitis. Med Dosw Mikrobiol 1993;45(2):163-6.

18. Kaur K, Prabha V. Spermagglutinating Escherichia coli and its role in infertility: in vivo stud. Microb Pathog 2014:69-70,33-8.

19. Merino G, Carranza-Lira S, Murrieta S, Rodriguez L, Cuevas E, Morán C. Bacterial infection and semen characteristics in infertile men. Arch Androl. 1995 Jul-Aug; 35(1):43-7.

20. Szponar M, Galiński J. Group B streptococci as an etiologic agent of infections in adults and their characteristics. Fertil Steril. 2014 Sep;102(3): 711-719.

21. Verani JR, McGee L, Schrag SJ; Prevention of perinatal group B streptococcal disease--revised guidelines from CDC, 2010. Division of Bacterial Diseases, National Center for Immunization and Respiratory Diseases, Centers for Disease Control and Prevention (CDC). MMWR Recomm Rep. 2010; 59:1-36.

22. Moretti E, Figura N, Collodel G, Ponzetto A. Can Helicobacter pylori infection influence human reproduction? World J Gastroenterol 2014; 20(19): 5567-74

23. Al-Sweih NA, Al-Fadli AH, Omu AE, Rotimi VO. Prevalence of Chlamydia trachomatis, Mycoplasma hominis, Mycoplasma genitalium, and Ureaplasma urealyticum infections and seminal quality in infertile and fertile men in Kuwait. Androl 2012; 33(6):1323-9.

24. Mackern-Oberti JP, Motrich RD, Breser ML, Sánchez LR, Cuffini C, Rivero VE. Chlamydia trachomatis infection of the male genital tract: an update. J Reprod Immunol 2013; 100 (1):37-53.

25. Recommendations for the Laboratory-Based Detection of Chlamydia trachomatis and Neisseria gonorrhoeae, Recommendations and Reports, Morbidity and Mortality Weekly Report (MMWR) March 14, 2014 / 63 (RR02); 1-19.

26. Abusarah EA, Awwad ZM, Charvalos E, Shehabi AA. Molecular detection of potential sexually transmitted pathogens in semen and urine specimens of infertile and fertile males. Diagn Microbiol Infect Dis 2013; 77 (4): 283-6.

27. Mehta RH, Sridhar H, Vijay Kumar BR, Anand Kumar TC. High incidence of oligozoospermia and teratozoospermia in human semen infected 
with the aerobic bacterium Streptococcus faecalis. Reprod Biomed Online 2002; 5(1):17-21.

28. EUR Lex 38-40 COMMISSION DIRECTIVE 2006/17/EC L38/40, of 8 Febr 2006, implementing Directive 2004/23/EC of the European Parliament and of the Counsil as regards certain Received 2-1-2018 technical requirements for the donation procureRevised 22-1-2018 ment and testing of human tissues and cells. 